\title{
SUPERFÍCIE FOLIAR DE GOMIDESIA SPECTABILIS (DC.) BERG E G. NITIDA (VELL.) LEGR. (MYRTACEAE) ${ }^{1}$
}

Recebido em 18.06.93. Aceito em 29.09.95.

\author{
Dória Maria Saiter Gomes ${ }^{2}$ \\ Raul Dodsworth Machado ${ }^{3}$
}

RESUMO: (Superfície foliar de Gomidesia spectabilis [DC.] Berg e G. nitida [Vell.] Legr. [Myrtaceae]) São apresentados dados relativos a micromorfologia da superfície foliar de duas espécies de Gomidesia (Myrtaceae). O estudo foi realizado em indivíduos que ocorrem na Floresta da Tijuca (Mata Atlântica), Rio de Janeiro. Atenção especial é dada ao tipo de cera epicuticular, relevo, estômatos e tricomas. Características peculiares são observadas nos tricomas de $G$. spectabilis.

Palavras-chave: MEV, Gomidesia, Myrtaceae

ABSTRACT: (Leaf surface of Gomidesia spectabilis [DC.] Berg and G. nitida [Vell.] Legr. [Myrtaceae]). Micromorphology characteristics of leaf surface of Gomidesia specimens are presented. The material studied was collected from plants which grow in Floresta da Tijuca (Mata Atlântica), Rio de Janeiro municipality. Special focusing is concentrated on the epicuticular wax type, relief, stomata and trichomes. The last ones show peculiar aspects in G. spectabilis.

Key words: SEM, Gomidesia, Myrtaceae

\section{Introdução}

O parque Nacional da Tijuca compreende uma vegetação típica de mata pluvial tropical, encerrando um grande número de espécies,constituindo um dos últimos remanescentes da Mata Atlântica do estado do Rio de Janeiro, região fitogeográfica que vem sofrendo ação antrópica progressiva.

A mata é composta por numerosas famílias botânicas destacando-se entre elas, as Myrtaceae.

1 Trabalho financiado parcialmente pelo CNPq.

2 Departamento de Botânica, Instituto de Biologia, Universidade Federal do Rio de Janeiro, Centro de Ciências da Saúde, Bloco A, Ilha do Fundão, Cidade Universitária, 21949-900, Rio de Janeiro, RJ, Brasil.

${ }^{3}$ Laboratório Hertha Mayer, Instituto de Biofísica Carlos Chagas Filho, Universidade Federal do Rio de Janeiro, Centro de Ciências da Saúde, Bloco G, Ilha do Fundão, Cidade Universitária, 21949-900, Rio de Janeiro, RJ, Brasil. 
A família Myrtaceae compreende cerca de 3500 espécies, subordinadas a aproximadamente 100 gêneros, distribuídos pela América Tropical e Austrália (Barroso et al. 1984). O gênero Gomidesia compreende 43 espécies (Legrand 1958) distribuídos pelo Brasil Central e Austral, alcançando o Nordeste com apenas uma espécie comum ao Brasil e as Antilhas (Legrand \& Klein 1967).

Entre os representantes do gênero ocorrentes no Maciço da Tijuca, figuram Gomidesia spectabilis (DC.)Berg e Gomidesia nitida (Vell.)Legr.

Na pesquisa bibliográfica, encontramos poucas informações sobre a superfície foliar da família Myrtaceae, sobretudo no que diz respeito a microscopia eletrônica de varredura. Machado et al. (1988) estudaram a anatomia da folha de Eugenia sulcata Spring ex Mart., enfocando aspectos micromorfológicos. Fontenelle et al. (1993) descreveram a superfície foliar de duas espécies de Gomidesia de restinga. Além disso, trabalhos como os de Carr et al. (1971), Johnson (1980), Van Wyk et al. (1982) e Bisse \& Rodriguez (1983), com os gêneros Eucalyptus L'herit, Leptospermun Forst., Eugenia L., Psidium L., e Myrtus L., respectivamente, destacam a importância da micromorfologia na resolução de problemas taxonômicos da família Myrtaceae.

Tendo em vista que informações sobre a micromorfologia foliar da família Myrtaceae são pouco numerosas, o presente trabalho tem como objetivo o estudo da superfície das folhas de Gomidesia spectabilis (DC.) Berg e Gomidesia nitida (Vell.) Legr., visando contribuir para o melhor conhecimento da família Myrtaceae e das espécies que ocorrem na Mata Atlântica.

\section{Material e métodos}

O material utilizado no presente estudo é proveniente da Floresta da Tijuca (Mata Atlântica), município do Rio de Janeiro. G. spectabilis foi coletada na estrada da Vista Chinesa, Km 1, cuja altitude é de aproximadamente $360 \mathrm{~m}$, enquanto $G$. nitida foi coletada no Morro do Queimado, $\mathrm{com}^{+}$. $550 \mathrm{~m}$ de altitude, vertente sul, ambos no Alto da Boa Vista.

Exemplares representativos das espécies encontram-se depositados no herbário do Departamento de Botânica da UFRJ, sob os números RFA 23371 e 23372 para G.spectabilis, e RFA 23467 para G.nitida.

Para exame ao microscópio eletrônico de varredura (MEV), foram utilizados fragmentos de folhas adultas herborizadas. A metade do material foi fervido em clorofórmio puro, com a finalidade de remover a cera superficial (Martin \& Juniper 1970). A outra parte foi mantida intacta. Os materiais tratados e não tratados foram montados em suporte adequado,e receberam uma cobertura de ouro para observação posterior. Para facilitar a análise,hifas de fungo foram removidas empregando-se a seguinte técnica: os fragmentos das folhas foram lavados em detergente Teepol por cinco minutos,colocados em água e submetidos a ultrasom por três minutos. A seguir foram lavados em álcool $70^{\circ} \mathrm{GL}$ e $100^{\circ} \mathrm{GL}$ por quinze minutos em cada, secos ao ar livre e montados em suporte. 
As eletromicrografias foram obtidas ao microscópio eletrônico de varredura Jeol 25-S-II.

\section{Resultados}

Nas espécies estudadas, verificou-se a presença de cera epicuticular, que pode ser de três tipos: grânulos, plaquetas e camada contínua. O tipo grânulo ocorre em ambas as epidermes de G. spectabilis (Figuras 3, 8 e 9) e na face abaxial de G. nitida (Figura 16). Estes grânulos possuem tamanhos variados e distribuem-se irregularmente pela superfície foliar, estando algumas áreas desprovidas de ornamentação de cera, inclusive sobre as células estomáticas (Figura 3). Na epiderme adaxial de G. nitida, a cera epicuticular deposita-se de maneira contínua. Por sobre essa camada contínua ocorre ainda o tipo plaqueta, distribuído regularmente sobre a superfície (Figuras 17 e 18).

A análise da epiderme adaxial, revelou cutícula proeminente ao nível das paredes anticlinais das células epidérmicas conferindo um aspecto reticulado à superficie foliar (Figuras 1, 2 e 10). Em G. nitida, verificou-se também a presença de estrias epicuticulares (Figura 10).

A epiderme abaxial revelou cutícula ligeiramente elevada, principalmente nas proximidades dos estômatos (Figura 3), e estriada ao nível das nervuras de G. spectabilis (Figura 5). Em G. nitida, a cutícula apresenta-se elevada sobre as paredes periclinais, conferindo um aspecto papiloso a esta superfície (Figura 12). Os estômatos estão localizados ligeiramente acima das demais células epidérmicas (Figuras 3, 11 e 12). Em G.nitida verificou-se um enrugamento da cutícula dos elementos epidérmicos que circundam as células estomáticas (Figuras 11 e 13). A presença de um halo claro ao redor do poro estomático, corresponde a uma projeção cuticular a este nível (Figura 3).

Em ambas as espécies ocorrem numerosos tricomas. Estes, são unicelulares, com formas e tamanhos variados. Foram observados dois tipos básicos nas espécies em questão: o primeiro, tipo I, é alongado (Figuras 1, 2, 4, 13 e 14) e frequentes vezes assume a configuração de bigorna (Figura 2). O segundo, tipo II, de forma "sui generis" (Figuras 4 a 7) é curto, com projeções aleatórias, arredondadas, que fornecem a estes tricomas diversos aspectos, destacando-se em muitos uma extremidade afilada. O tipo I, ocorre em ambas as faces da folha de G. spectabilis (Figuras 1 e 4), sendo mais abundante na adaxial, e na face abaxial de G. nitida (Figura 13). Nesta espécie, estão dispostos inclinadamente, cobrindo totalmente a superfície foliar (Figura 14), e são frequentemente providos de uma ornamentação estriada (Figura 15). Ao cairem, deixam na epiderme, uma cicatriz caracterizada pela disposição radial dos demais elementos epidérmicos (Figura 11). O tipo II, ocorre somente na epiderme abaxial de G. spectabilis (Figuras 4 e 7), estando em maior número sobre as nervuras (Figura 4). A epiderme adaxial de $G$. nitida é glabra.

Numerosas hifas de fungo ocorrem em ambas as epidermes das duas espécies analisadas (Figuras 6, 8 e 14). 

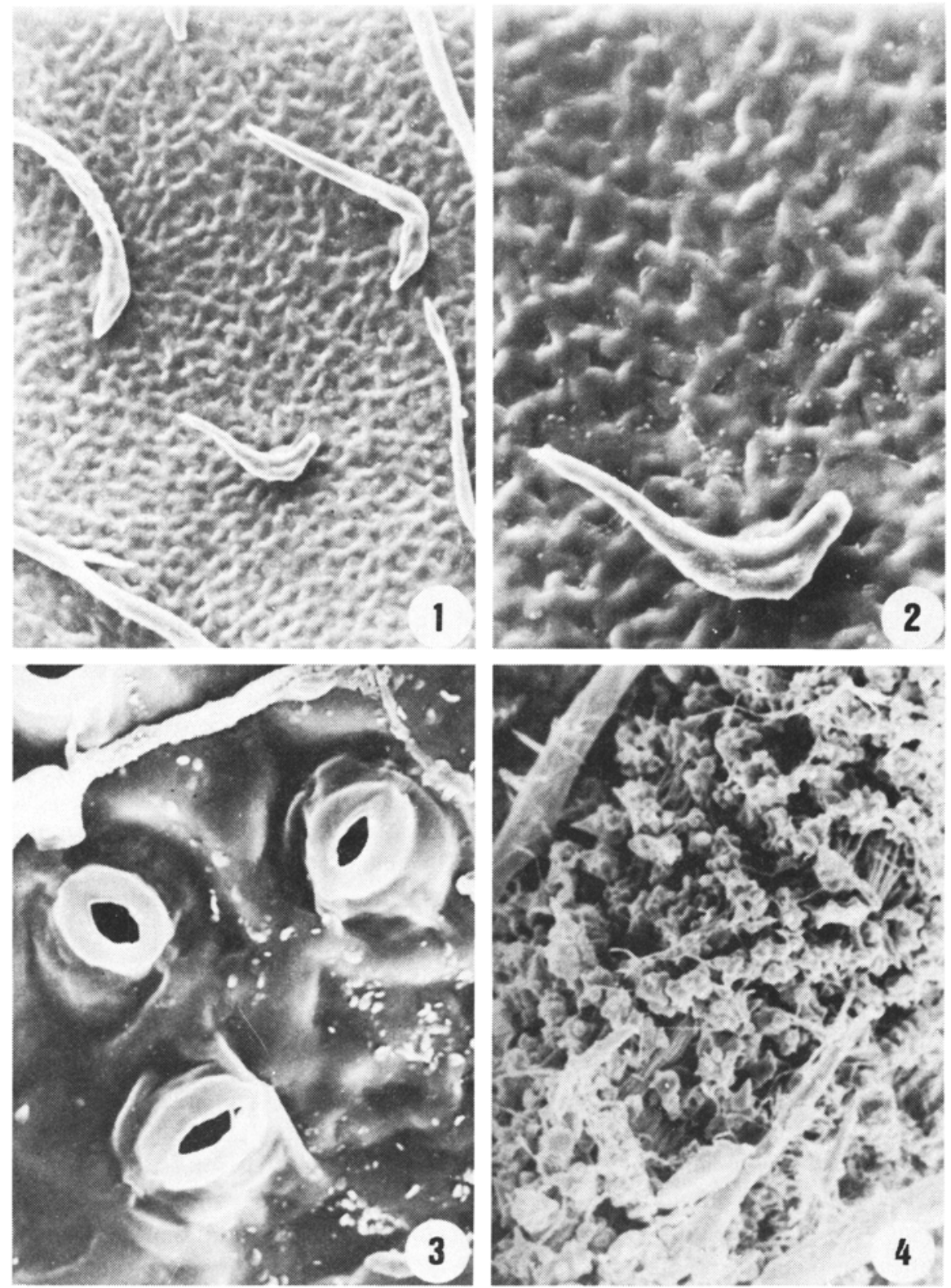

Figuras 1-4. Superfície foliar de G. spectabilis. 1. Epiderme adaxial evidenciando superfície reticulada e tricomas unicelulares simples $(300 \mathrm{x})$. 2. Epiderme adaxial mostrando tricoma em forma de bigorna (700) x). 3. Epiderme abaxial com estômatos $(2000 \mathrm{x})$. 4. Epiderme abaxial sobre nervura com numerosos tricomas do tipo II $(200 \mathrm{x})$. 


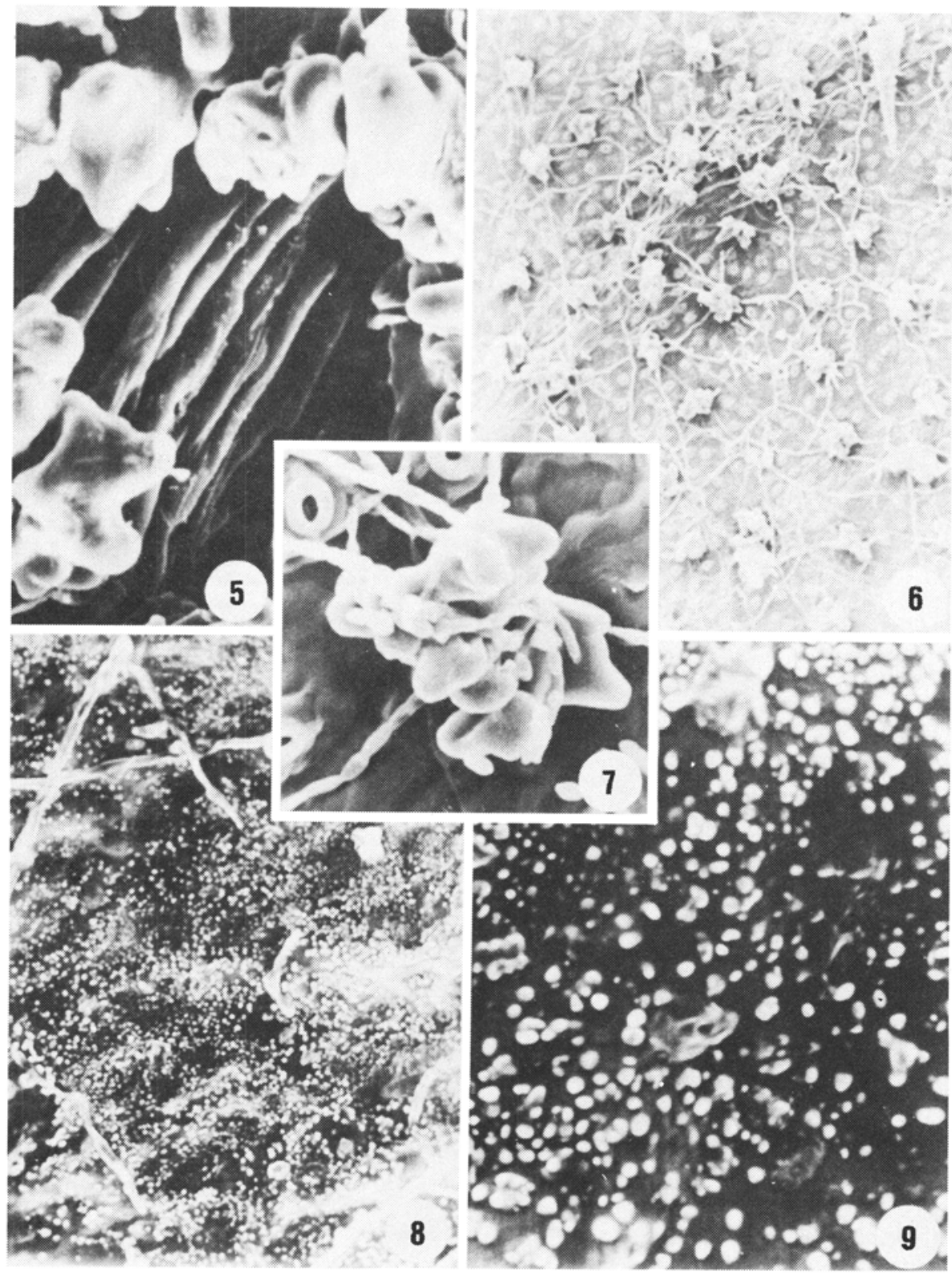

Figuras 5-9. Superfície foliar de G. spectabilis. 5. Epiderme abaxial mostrando cutícula estriada sobre nervura $(1000 \mathrm{x}) .6$. Aspecto geral da epiderme abaxial, evidenciando estômatos, tricomas do tipo II e numerosas hifas de fungos $(150 \mathrm{x}) .7$. Detalhe de um tipo de tricoma "sui generis" (1000 x). 8. Aspecto geral da epiderme adaxial mostrando cera em grânulos $(700 \mathrm{x}) .9$. Detalhe da cera epicuticular da face adaxial $(3000 \mathrm{x})$. 

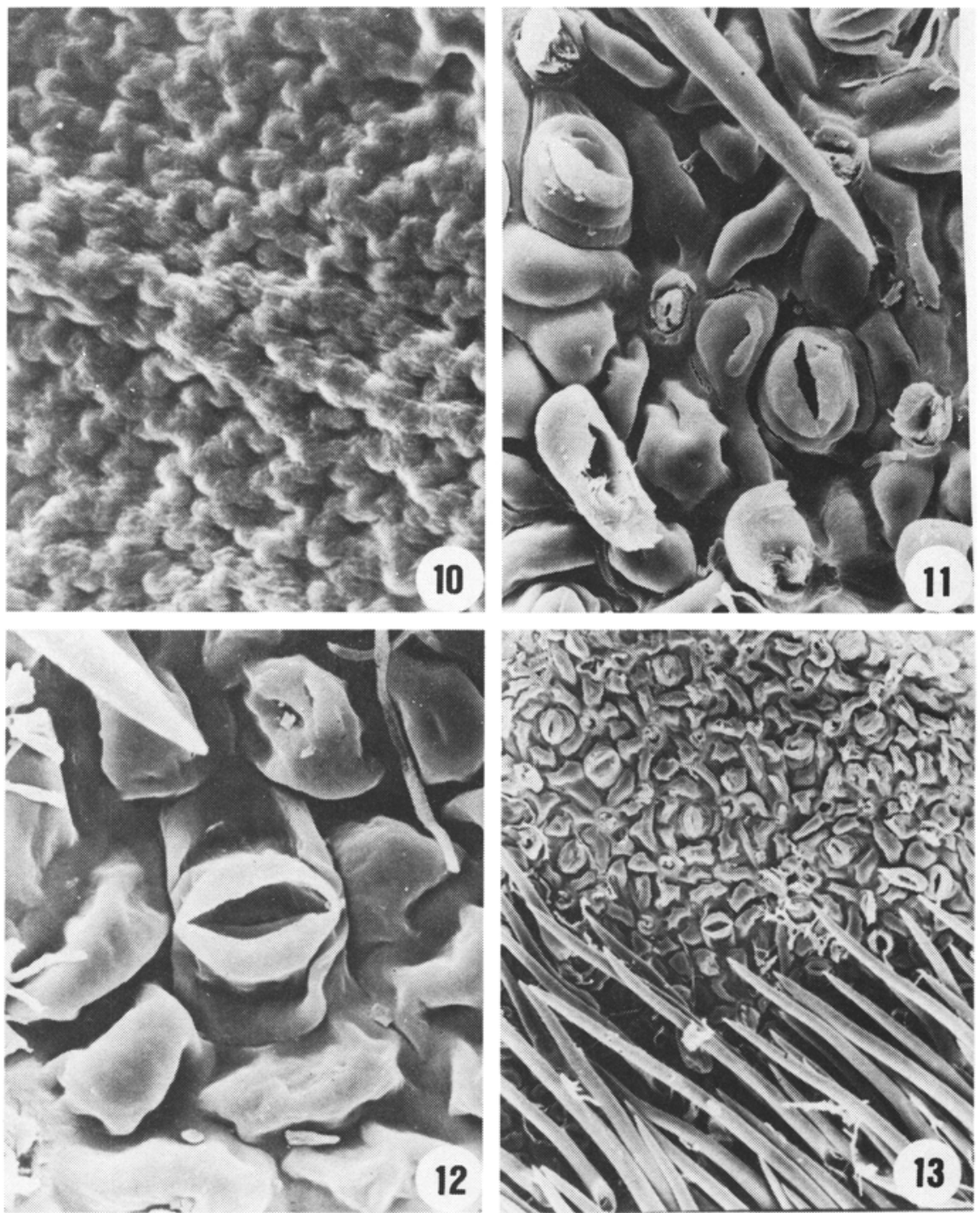

Figuras 10-13. Superfície foliar de G. nitida. 10. Epiderme adaxial $(700 \mathrm{x})$. 11. Epiderme adaxial mostrando estômatos e cicatriz deixada por um tricoma $(1000 \mathrm{x})$. 12. Detalhe da epiderme abaxial. Note papilas e estômato $(1500 x) 13$. Aspecto geral da epiderme abaxial evidenciando numerosos tricomas e uma região onde os mesmos foram retirados $(300 \mathrm{x})$. 


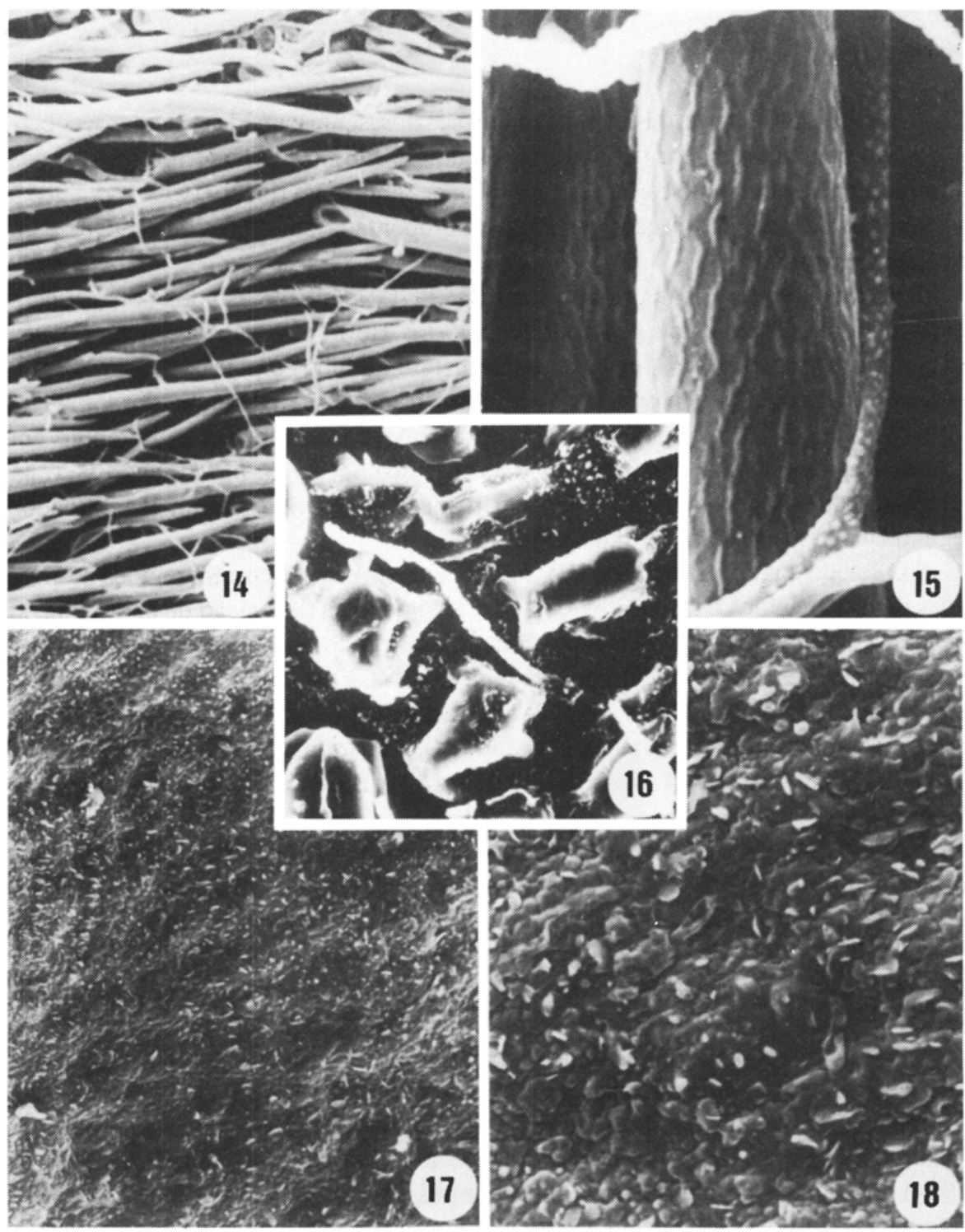

Figuras 14-18. Superfície foliar de G. nitida. 14. Epiderme abaxial com densa cobertura de pêlos (200 x). 15. Detalhe de um pêlo mostrando ornamentação estriada e hifas de fungos $(4500 \mathrm{x})$. 16. Epiderme abaxial com cera em grânulos $(1500$ x). 17. Aspecto geral da epiderme adaxial evidenciando cera em plaquetas (700 x). 18. Detalhe de cera epicuticular da face adaxial $(2000 \mathrm{x})$. 


\section{Discussão}

A superfície externa das plantas superiores apresenta camada cuticular recoberta por um depósito de cera (Eglinton \& Hamilton 1967), que pode formar padrões definidos, utilizados como caráter taxonômico na família Myrtaceae (Carr et al. 1985). A excreção de cera nos órgãos vegetativos é apontada por Solereder (1908) para espécies de Myrtaceae.

Hallam (1970) e Hallam \& Chambers (1970) em seus estudos sobre a cera epicuticular em espécies do gênero Eucalyptus L'Herit, afirmam que ocorrem três tipos básicos de cera: tubos, placas e uma mistura de tubos e placas. Segundo estes autores o ambiente pode interferir no tipo e no arranjo da cera epicuticular.

Nas espécies em questão, verifica-se a presença de cera do tipo grânulo em ambas as epidermes de $G$. spectabilis e na face abaxial de $G$. nitida, ocorrendo na epiderme adaxial desta última o tipo em plaqueta. Tais tipos foram identificados segundo a classificação de Wilkinson (1979).

O estudo das epidermes ao MEV também mostrou que ocorrem regiões desprovidas de ornamentação. Nestas regiões, provavelmente, a deposição de cera se dá de maneira contínua sobre a superfície foliar,que segundo Barthlott \& Wollenweber (1981) parece ser o caso mais frequente para as plantas vasculares.

Na epiderme adaxial de G. nitida a cutícula é estriada, assim como nas células epidérmicas sobre nervuras da face abaxial de G. spectabilis. Tais estriações são semelhantes às descritas por Johnson (1980) para várias espécies de Leptospermum Forst.

As papilas encontradas na epiderme abaxial de G. nitida são conspícuas, regularmente distribuídas pela superfície foliar e caracterizadas pela microscopia eletrônica de varredura como pequenas estruturas globulares. Segundo Johnson (1980), a ocorrência de papilas em Leptospermum coriaceum e L. laevigatum é constante, sendo que nesta última espécie, as papilas apresentam ornamentação em seu ápice.

Ladiges (1984) faz um estudo comparativo de tricomas de Angophora Cav. e Eucalyptus L'Herit, enfocando aspectos evolutivos. Dentre os tipos descritos, destacam-se pêlos glandulares, cuja forma se assemelha aos encontrados na epiderme abaxial de G. spectabilis. Analisando a superfície dos pêlos glandulares, Ladiges (1984) relata que a mesma é ornamentada por três ou mais micropapilas. Tricomas de cutícula lisa e forma semelhante aos de G. spectabilis, são também referidos para a epiderme de G. martiana Berg. por Fontenelle et al. (1993).

Em G. nitida, quando os pêlos são observados ao MEV, é possível notar uma ornamentação estriada em sua superfície. Entretanto, na bibliografia consultada, não foi encontrada referência para este tipo de ornamentação em tricomas da família Myrtaceae. 


\section{Referências bibliográficas}

Barroso, G.M.; Peixoto, A.L.; Ichaso, C.L.F.; Costa, C.G.; Guimar es,E.F. \& Lima, H.C. 1984. Sistemática de Angiospermas do Brasil. Viçosa, UFV, Imp. Univ., v. 2, 377p.

Barthlott, W. \& Wollenweber, E. 1981. Zur Feinstruktur, Chemie und taxonomischen signifikanz epicuticularer Wachse und Ahnlicher sekrete. Tropische und subtropische Pflanzewelt, 32:1-67.

Bisse, J. \& Rodriguez, R.R. 1983. Comparación morfoanatomica de los géneros Psidium L y Myrtus L. (Myrtaceae) en Cuba. Revista del Jardim Botanico, 4(3):11-26.

Carr, S.G.M.; Milkovits, L. \& Carr, D.J. 1971. Eucalypt Phytoglyphs: The microanatomical features of the epidermis in relation to taxonomy. Aust. J. Bot., 19:173-19O.

Carr, D.J.; Carr, S.G.M. \& Lenz, J.R. 1985. Oriented arrays of epicuticular wax plates in Eucalyptus species. Protoplasma, 124:205-212.

Eglinton, G. \& Hamilton, R. 1967. Leaf epicuticular waxes. Science, 156(378O):1322-1335.

Fontenelle, G.B.; Gomes, D.M.S. \& Machado, R.D. 1993. Anatomia foliar de Gomidesia martiana Berg. e G. fenzliana Berg. (Myrtaceae). Revta. bras. bot., 16 (1): 17-30

Hallam, N.D. 1970. Growth and regenerative of waxes on the leaves of Eucalyptus. Planta, 93:257-268.

Hallam, N.D. \& Chambers, T.C. 197O. The leaf waxes of the genus Eucalyptus L'Hérit. Aust. J. Bot., 18:335-386.

Johnson, C.T. 1980. The leaf anatomy of Leptospermum Forst. (Myrtaceae). Aust. J. Bot., 28:77-1O4.

Ladiges, P.Y. 1984. A comparative study of trichomes in Angophora Cav. and Eucalyptus L'Hérit.- a question of homology. Aust. J. Bot., 32:561-574.

Legrand, C.D. 1958. Las especies tropicales del genero Gomidesia. Comun. Bot. Mus. Hist. Nat. Montev., 3(37):1-3O.

Legrand, C.D. \& Klein, R.M. 1967. Mirtáceas 1- Gomidesia Berg. Flora Ilustrada Catarinense I. Parte: As plantas, fasc. Mirt. 1-44.

Machado, R.D.; Costa, C.G. \& Fontenelle, G.B.. 1988. Anatomia foliar de Eugenia sulcata Spring ex Mart. (Myrtaceae). Acta Bot. Bras., l(2)supl.:275-285.

Martin, J.T. \& Juniper, B.E. 197O. The Cuticles of Plants. Edinburgh, Edward Arnold Ltd., 347p.

Solereder, H. 1908. Systematic anatomy of the dicotyledons. Oxford, Claredon Press, 1182p.

Van Wyk, A.E.; Robbertse, P.J. \& Kok, P.D.F. 1982. The genus Eugenia L. (Myrtaceae) in southern Africa: The structure and taxonomic value of stomata. J. Linn. Soc. (Bot.), 84:41-56.

Wilkinson, H.P. 1979. The plant surface. In: Metcalfe, C.R. and Chalk, L. Anatomy of the dicotyledons. Oxford, Claredon Press, 1:97-165. 